\title{
Diet selection by cattle in an extended grazing season system
}

\author{
P. D'hour, B. Dumont
}

Laboratoire adaptation des herbivores aux milieux, Inra, 63122 Saint-Genès-Champanelle, France

When grazing pressure is low, reproductive patches appear in pastures and are largely avoided because animals prefer vegetative regrowths. Extending the grazing season could limit the accumulation of dead material in pastures. We observed the choice of eight mature Salers cows in a 12 -ha natural mountain pasture (1150 m, main species: Agrostis tenuis, Festuca rubra) i) in mid-April before the grass started to grow (green material: $5.5-\mathrm{cm}$ high, $59 \%$ of area); ii) in late-August (green material: $9.4 \mathrm{~cm}, 76 \%$ ); and iii) in December 2 months after the end of the growing season (green material: $4.0 \mathrm{~cm}, 51 \%$ ). Twelve 10 -min recordings were made per animal in each period. Bites were separated into green (GB: $>90 \%$ of green material), dry (DB: $>90 \%$ of dead material) and mixed bites. Nitrogen content of faeces was analysed. Compared to late-August ( $95 \% \mathrm{~GB}, 2.2 \% \mathrm{~N}$ in faeces), cows were more willing to consume dead material out of the traditional grazing season $(P<0.001)$. They selected less GB in December than in April (29 versus 78\%; $P<0.001$ ) although height and proportion of vegetative areas were similar. In December, cows also selected $7 \%$ DB vs $0.3 \%$ in April $(P<0.001)$. Faeces nitrogen content was consistently lower in December ( 1.6 vs $2.3 \% ; P<0.001$ ). Grazing in early spring limited grass growth and consequently the accumulation of dead material during the summer, but it is clearly in the autumn that cows switched to the poor-quality patches.

\section{Pâturage hivernal des brebis : production fourragère et performances animales}

\author{
E. Pottier, L. Sagot \\ Institut de l'élevage, ferme expérimentale ovine, Le Mourier, 87800 Saint-Priest Ligoure, \\ France
}

Avec l'agrandissement des exploitations, le pâturage hivernal de brebis vides ou gestantes doit permettre de limiter les besoins en bâtiments, de réduire les contraintes de travail et de mieux gérer la pousse printanière de l'herbe. Sa mise en ouvre appelle la mise au point de règles de pilotage des surfaces pour préserver l'équilibre entre fauche et pâture en assurant des performances animales satisfaisantes. Dans ce contexte, nous avons comparé la production fourragère de deux parcelles de raygrass anglais, chacune d'elles étant divisée de façon pérenne en deux paddocks pâturés ou non en hiver. Par ailleurs, nous avons comparé les besoins et les performances de brebis conduites en pâturage hivernal tournant sur 17 parcelles à celles de brebis témoins hivernant en bergerie, Le pâturage en janvier puis février n'a pas affecté la croissance de l'herbe au printemps. Cependant, la différence de quantité d'herbe disponible à l'issue de la dernière exploitation hivernale s'est maintenue au cours du premier cycle essentiellement. L'herbe valorisée en hiver correspond aux stocks non pâturés à l'automne (dans la strate $2-3 \mathrm{~cm}$ à l'herbomètre) et à une pousse hivernale plus importante sur les paddocks pâturés ( $380 \mathrm{vs.} 280 \mathrm{~kg} \mathrm{MS} \cdot h^{-1}$ en février). Les faibles hauteurs d'herbe n'ont pas pénalisé l'ingestion, estimée à $1,4 \mathrm{~kg} \mathrm{MS} / \mathrm{j} /$ brebis. Finalement, les besoins en fourrages récoltés des brebis maintenues au pâturage en hiver ont été faibles : $6 \mathrm{vs.} 69 \mathrm{~kg} \mathrm{MS} / \mathrm{brebis}$, mais leurs besoins en surface pâturée ont été élevés : 25 ares/brebis. Les conséquences sur la pérennité du couvert végétal sont à l'étude. 